\title{
Development of pediatric academic-community radiology services: lessons learned
}

\author{
Victor J. Seghers ${ }^{1}$ - Andrew C. Sher ${ }^{1}$ • HaiThuy N. Nguyen ${ }^{1}$ • Shireen E. Hayatghaibi ${ }^{1}$. \\ Thierry A. G. M. Huisman ${ }^{1}$ • Marla B. K. Sammer ${ }^{1}$
}

Received: 22 November 2019 / Revised: 12 February 2020 / Accepted: 12 March 2020 / Published online: 2 May 2020

(C) Springer-Verlag GmbH Germany, part of Springer Nature 2020

\section{Introduction}

Academic medical centers are expanding services in community locations [1,2], thereby improving patient access to care in new geographic regions and reducing disparity of care by offering subspecialty services to historically underserved populations. While the recent expansion of adult academic medical centers into community locations has been described, including community expansion models focused on academic radiology [3-8], there has not been a similar focus on the developing community-academic partnerships in the pediatric sphere.

The academic medical center tripartite mission of education, clinical care and research is expensive, costing 10-20\% more than non-academic medical centers on a case-mix adjusted basis [9, 10]. Additionally, academic medical centers have been disproportionately affected economically by health care reform initiatives in the United States designed to improve quality through reduced payments to low-performing hospitals that may not adequately risk-adjust for differences in case mix, patient comorbidities and patient sociodemographic characteristics $[2,9,11]$. The prior academic model was financially sustainable in part because of external research grant funding, governmental and institutional subsidies, philanthropic donations, lower salaries relative to private practice, and favorable reimbursements [2]. Compared to the 1980s, when clinical services represented only $20 \%$ of academic medical center revenues, currently they account for nearly $80 \%$ of total revenues [1].

In response to these economic pressures, academic centers are expanding into the community in a variety of ways - via merger, acquisition, partnerships with existing community

Victor J. Seghers

vjsegher@texaschildrens.org

1 Edward B. Singleton Department of Radiology, Texas Children's Hospital and Baylor College of Medicine, 6701 Fannin St., Suite 470, Houston, TX 77030, USA health systems, or by a "brick-and-mortar" approach in which they physically expand to new locations $[1,2]$. These models vary by amount of up-front capital investment, time to profitability, and need to develop referral relationships and brand impact via their ability to control and integrate employees, resources, processes and culture. Economies of scale can be attained by serving a larger patient population without a full capital investment in the community location, which decreases cost per procedure, and by channeling patients from the community to the main academic center who require advanced levels of care, which improves asset utilization and productivity [2].

Pediatric academic health systems, facing the same economic pressures, are increasingly developing a community strategy. Here, we discuss the opportunities and challenges experienced over the last decade in creating a pediatric academic-community radiology practice within a large pediatric academic health system.

\section{Historical context}

Texas Children's Hospital (TCH) is a quaternary care pediatric academic health system based in Houston, TX. The system includes its primary teaching hospital in the Texas Medical Center (TCH-TMC); Texas Children's-Pavilion for Women, an adult hospital that focuses on women, mothers and babies; as well as two community pediatric hospitals, TCH-West Campus and TCH-The Woodlands, which opened for inpatient care in 2011 and 2017, respectively. There are also 7 specialty care centers and 11 urgent care centers at community locations throughout the metro-Houston region (Fig. 1). Texas Children's operates all of its hospitals under a single Tax ID, allowing physicians to be credentialed only once to provide care at all its locations. Consequently, this provides significant flexibility in radiologist staffing. 
Fig. 1 Texas Children's Hospital (TCH) community locations throughout the Houston metropolitan region. Red diamonds designate the three pediatric hospitals and green dots designate specialty care centers. TCH-West Campus and TCHThe Woodlands are 23 miles and 38 miles away, respectively, from TCH-Texas Medical Center. TCH-West Campus and TCHThe Woodlands are 45 miles apart

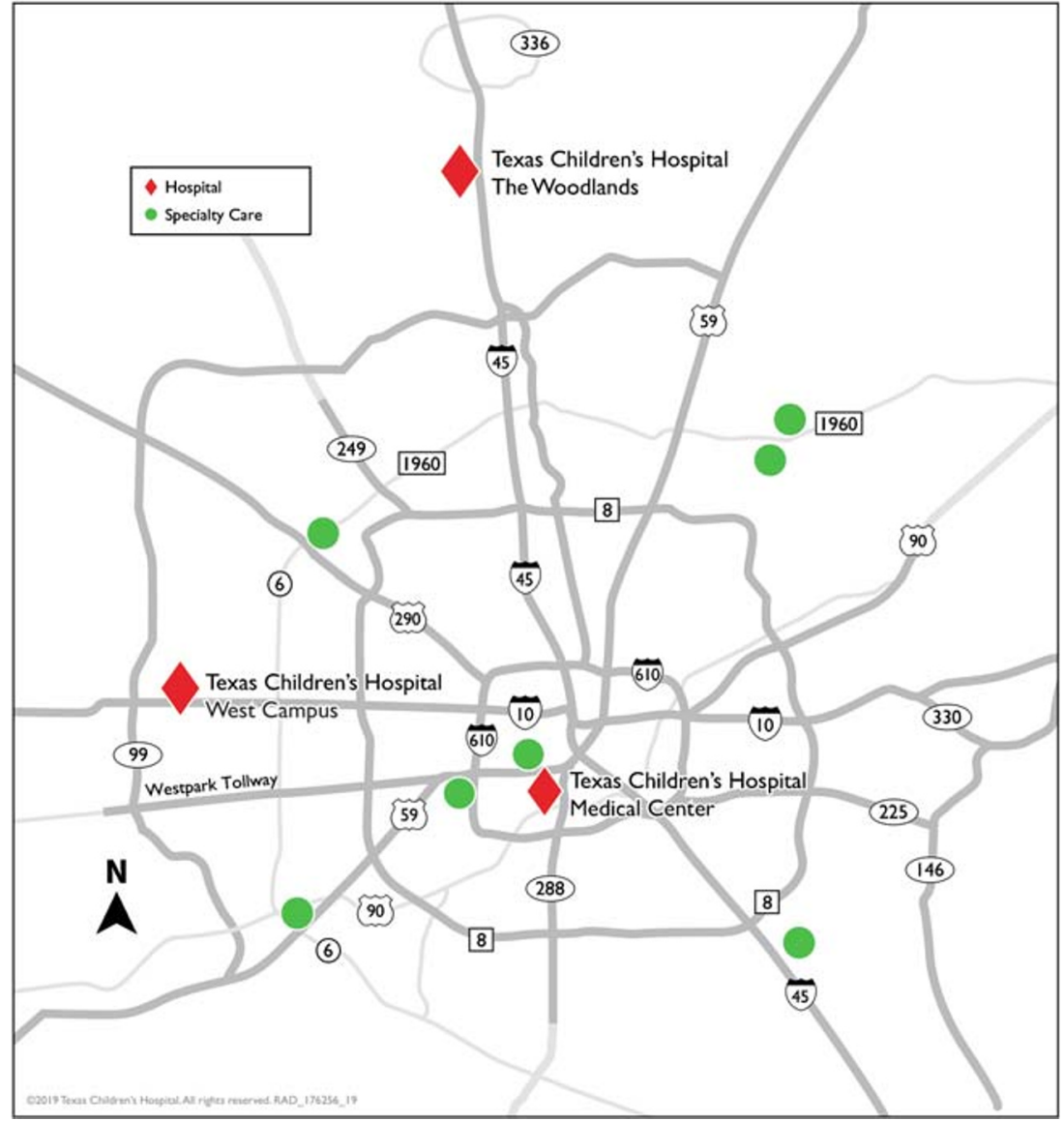

Inclusive of the TCH-Pavilion for Women, which is physically attached to the main teaching hospital, TCH has a total of 994 licensed beds (TCH-TMC: 834, TCH-West Campus: 86, TCH-The Woodlands: 74). Both community pediatric hospitals are full-service hospitals with inpatient and outpatient services, pediatric intensive care unit (ICU), emergency center and operating suites. TCH-The Woodlands also offers a Level 3 neonatal ICU. Some services, including neurosurgery, cardiac surgery, high-acuity trauma and transplant services, are outside the scope of care for the community hospitals.

The radiology departments at both community hospitals offer ultrasound (US), CT, MRI, fluoroscopy, interventional radiology and radiography, with current relative modality volumes described in Fig. 2. Nuclear medicine services are only offered at TCH-TMC and not in the community. In-house sonographers, CT and radiography technologists are available $24 \mathrm{~h}$ a day, 7 days a week (24/7), with MRI and interventional radiology services available after hours on an emergent basis. Child life specialists are present at all campuses. Anesthesia is available for sedation 24/7, with beeper call for emergent cases at all campuses. Depending on location, the specialty care centers and urgent care centers offer pediatric ultrasound or radiography imaging services.

Expansion into community sites was part of a system-wide initiative to improve and extend pediatric clinical care in the
Houston metropolitan region. We developed a coordinated strategy based on population demographics, migration patterns and historical patient patterns such as home ZIP codes of existing patients. Under the direction of our $\mathrm{TCH}$ Marketing Department, a campaign comprising advertising in local media, sponsorship of community events, and faceto-face contact with both $\mathrm{TCH}$ and non-TCH pediatric practices was created to educate the community regarding improved access to high-quality pediatric care as well as to develop referral relationships. Institution administrators included radiology leadership in discussions of the types of imaging modalities to provide, equipment selection, number of clinical full-time equivalents (FTEs) needed to support the projected imaging volume, and community radiology physician and administrative leadership recruitment. Additional staff hiring and equipment purchases were funded by the institution.

\section{Daily workflows}

Weekdays, each community hospital is staffed on-site by a pediatric body radiologist (7:30 am to $4: 30 \mathrm{pm})$ and an interventional radiologist (typically 7:00 am to 4:00 pm, but hours vary based on caseload). These rotations are primarily staffed by radiologists within the Community Radiology Division 
Fig. 2 Graph shows 2019 relative Texas Children's Hospital (TCH) Radiology Enterprise imaging volumes by modality and location

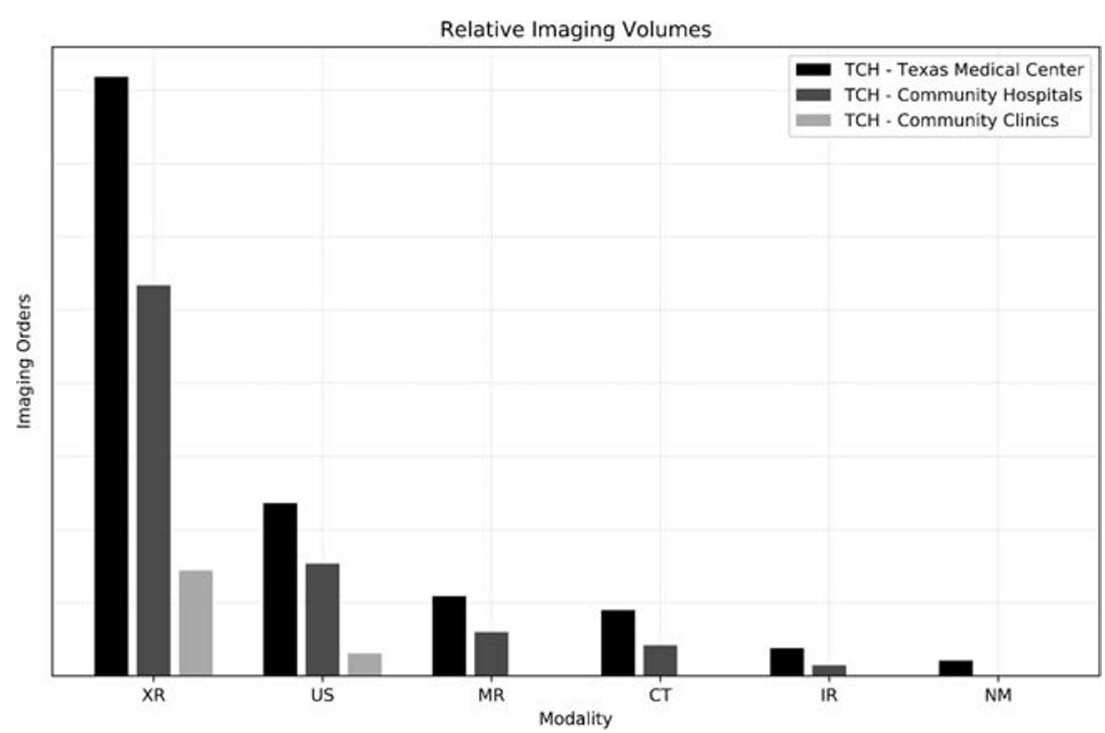

(currently 3 body and 1 interventional radiologist). When not available, body and interventional radiologists from TCMTMC staff the community rotations. Radiologists are not physically on-site at $\mathrm{TCH}$ urgent care or $\mathrm{TCH}$ specialty care centers, but they perform remote interpretation of imaging generated from those locations.

The Community Radiology Division was established in 2017 as a result of a systemwide vision of providing dedicated staffing to the community hospitals, with a primary intent to improve continuity of care and communications between providers and patients. Within radiology, the division between communitybased and medical-center-based radiologists is not strict, but, in general, community radiologists staff the community hospitals. However, members of the community division participate fully in weekend call rotations, which are in-house rotations at the Medical Center. As the schedule allows, any interventional or body radiologist can request to be scheduled at any location, if needed for professional or personal reasons.

Unlike at the academic center where rotations are modalityor systems-based (outpatient ultrasound, inpatient ultrasound, body CT/MR, fetal imaging, musculoskeletal imaging, neuroradiology, etc.), the community hospital rotations mimic a generalist approach. The daily on-site body radiologist is responsible for all modalities other than interventional procedures and cardiac, musculoskeletal and neuroradiology exams. Radiology trainees only rotate through TCH-West Campus and not TCH-The Woodlands because of the latter's greater distance from the academic medical center.

Outpatient imaging volumes far exceed inpatient volumes, and the bulk of the work for the on-site community radiologist involves radiography, ultrasound and fluoroscopy. Locally generated body CT and MRI are read by the on-site radiologist to expedite and improve clinical care at the request of referring providers, as well as to enable the community radiologists to maintain their interpretive skills, which they need when staffing after-hours rotations for the TCH system. The imaging modality with the largest volume is radiography, but this is the shared responsibility of the body radiologists across all campuses and read from a common work list, independent of the imaging acquisition location. All cardiac, musculoskeletal and neuroradiology coverage of the community hospitals is performed remotely from the main academic center, and this includes protocoling exams, reviewing imaging and reporting, and consultation.

Centralized scheduling is used throughout the TCH system to optimally schedule imaging procedures. The scheduling department uses a scripted workflow to determine where the exam can be performed, using rules that indicate procedures offered at each facility. These scheduling rules are frequently updated to reflect the expanding scope of community imaging. For example, MR enterography and cardiac CT were only offered at TCH-TMC and not at TCH-West Campus when it opened in 2011, but both modalities are now offered throughout the system because protocols were standardized, technologists were trained, and imaging equipment was upgraded. If an exam can be performed at multiple locations, the exam is scheduled based on the patient's location preference and earliest imaging appointment availability. Upcoming scheduled clinic appointments are also queried and coordinated for same-day appointments and same location when possible.

\section{Remote reading logistics}

Radiology studies of all modalities populate a single unified picture archiving and communication system (PACS; Philips Intellispace, Foster City, CA), which is maintained by the hospital system informatics department. An add-on PACS 
workflow orchestrator and communication solution (Prism; Nuance Communications, Burlington, MA) is also used to coordinate care and enable communication between radiologists and technologists across multiple sites. For example, TCH community specialty care centers are not staffed by a radiologist, so for ultrasound exams performed at these locations, the sonographer communicates his or her findings to the radiologist via a preliminary note that automatically opens in a pop-up window when the study is opened by any radiologist, regardless of location.

There are several layers of redundancy for communication across the TCH system, including instant messaging capability via the Prism communicator add-on within the PACS environment. For example, instant messaging is often used when a community hospital technologist needs a protocol for an add-on CT or MRI. Additionally, the phone number associated with the reading radiologist's PACS workstation automatically populates the communicator application, enabling technologists to easily call the physician for questions or to review exams such as ultrasounds. Finally, to more efficiently promote communication between radiologists and ordering providers throughout the TCH system, as well as address complex imaging rotation coverage rules that can make it difficult for technologists or ordering providers to contact the radiologist, a dedicated systemwide internal phone number (4RADS) was created. This is staffed $24 / 7$ by radiology personnel at TCH-TMC who are knowledgeable about rotational workflows and can direct questions to the correct rotation or radiologist.

\section{After-hours workflows}

Outside regular hours, community imaging is interpreted in real time by radiologists working in-house at the medical center academic hospital, allowing for $24 / 7$ coverage throughout the hospital system. For emergent after-hours interventional or fluoroscopy procedures, separate community interventional and community body radiology call pools exist that are independent from call responsibilities at the academic medical center. To address concerns from community clinical providers regarding unequal level of after-hours service at the academic center versus community hospitals, we established service guidelines that discuss types of procedures and time frames (Table 1).

All body radiology fluoroscopy services provided at TCHTMC are available at both community sites. After hours, these studies are primarily intussusception reductions and to a lesser extent upper gastrointestinal series for bilious emesis. For interventional radiology, procedures typically involve peripherally inserted central catheter (PICC) placement and abscess drainage and are limited largely by scope of surgical specialty care. For example, the community sites are not certified as
Level I trauma centers, and traumatic embolization cases would only be treated at TCH-TMC. Challenges with community call are discussed in more detail in the "Challenges and lessons learned" section.

\section{Super-specialty community imaging}

An essential part of our community imaging strategy is that all our locations offer the same high level of expertise, quality and safety. This commitment requires that imaging workflows and personnel at the community hospitals mimic those at the academic medical center, which can be challenging for superspecialty areas such as pediatric musculoskeletal, cardiac and interventional radiology.

Once per week a pediatric musculoskeletal radiologist from the academic center fulfills the system-wide musculoskeletal rotation on-site at each community hospital (total twice/week in the community), performing local musculoskeletal fluoroscopic procedures as well as interpreting cross-sectional imaging for the TCH system. Community musculoskeletal MR and fluoroscopic scheduling workflows have been enacted to promote efficiency and take advantage of on-site super-specialty clinical acumen. In an effort to improve access when the musculoskeletal radiologist is not on-site, interventional radiologists perform some musculoskeletal procedures at the community hospitals. Although not all interventional radiology (IR) physicians are trained in all the musculoskeletal procedures, when possible, the musculoskeletal radiologists include IR in the less frequent cases (such as treatment of ganglion cysts) to broaden their competency base. The improved communication and coverage has enabled other efficiencies, such as bundled procedures to enable joint aspirations after concerning MR findings to take advantage of a single sedation event.

Another opportunity for super-specialty care is in pediatric cardiac imaging. While there is not a dedicated cardiac imager on-site at the community hospitals, investments in state-ofthe-art scanners and education enable cardiac CT and MR to be performed at our community hospitals. The community CT and MR technologists have received additional training to enable quality performance of these exams, and they participate in system-wide quality reviews. The cardiac radiologist often supports the technologist over the telephone, viewing the images sent to the PACS in near real time and providing feedback while the patient is on the scanner and the study is being acquired.

The presence of pediatric interventional radiology at the community hospitals, including development of a multi-disciplinary vascular anomalies clinic at TCH-West Campus, further enables these community hospitals to be a place where care can come to the patient. Availability of interventional radiology services is a necessary support 
Table 1 Pediatric emergency fluoroscopy and interventional radiology service guidelines for add-on procedures

\begin{tabular}{|c|c|c|c|c|}
\hline $\begin{array}{l}\text { Time } \\
\text { Class }\end{array}$ & $\begin{array}{l}<2 \mathrm{~h} \\
\text { Emergent }\end{array}$ & $\begin{array}{l}<4 \mathrm{~h} \\
\text { Urgent }\end{array}$ & $\begin{array}{l}<12 \mathrm{~h} \\
\text { Semi-urgent }\end{array}$ & $\begin{array}{l}<24 \mathrm{~h} \\
\text { Done by next day }\end{array}$ \\
\hline Fluoroscopy & $\begin{array}{l}\text { Upper GI } \\
\text { Intussusception reduction }\end{array}$ & & & Naso-jejunal tube placement \\
\hline \multirow[t]{5}{*}{$\begin{array}{l}\text { Interventional } \\
\quad \text { radiology (IR) }\end{array}$} & $\begin{array}{l}\text { Any IR procedure indicated as } \\
\text { treatment of an unstable patient can } \\
\text { be requested (provider-provider } \\
\text { discussion on case-by-case basis) }\end{array}$ & Nephrostomy tube placement (septic) & $\begin{array}{l}\text { Fluid aspiration with } \\
\text { paracentesis, thoracentesis } \\
\text { (clinically symptomatic) }\end{array}$ & $\begin{array}{l}\text { Central venous access (including } \\
\text { PICC lines) }\end{array}$ \\
\hline & Angiogram (bleeding, unstable) & Abscess drainage (septic) & $\begin{array}{l}\text { Chest tube placement (clinically } \\
\text { symptomatic) }\end{array}$ & Abscess drainage (non-septic) \\
\hline & Arterial thrombolysis & $\begin{array}{l}\text { Cerebral angiogram (TCH-TMC } \\
\text { campus only) }\end{array}$ & Septic hip aspiration & $\begin{array}{l}\text { Gastro-jejunal tube } \\
\text { placement/replacement }\end{array}$ \\
\hline & $\begin{array}{l}\text { Life/limb-threatening venous } \\
\text { thrombolysis }\end{array}$ & Dialysis catheter placement & & Biopsy \\
\hline & Thrombolysis of pulmonary embolus & & & $\begin{array}{l}\text { Pheresis catheter } \\
\text { Lumbar puncture } \\
\text { Extremity venous thrombolysis } \\
\text { IVC filter placement }\end{array}$ \\
\hline
\end{tabular}

GI gastrointestinal, $h$ hours, IVC inferior vena cava, PICC peripherally inserted central catheter, TCH-TMC Texas Children's Hospital-Texas Medical Center

for the medical and surgical specialties and allows their growth within the community hospitals. It also prevents unnecessary transfers to the academic medical center. Offering high-quality super-specialty pediatric imaging services in the community is necessary if an academic pediatric medical center aims to compete with existing imaging providers and increase its clinical volume.

Pediatric neuroradiology is performed daily at the community hospitals and is interpreted exclusively by pediatric neuroradiologists based at the academic medical center. Upon opening the TCH-West Campus in 2011 and again upon opening TCH-The Woodlands in 2017, neuroradiologists staffed a community hospital once per week. Efforts were initiated to promote face-to-face consultation with TCH community-based neurologists as well as develop neurology clinical case conferences. Both of these initiatives failed, and it was hard to justify neuroradiologists based at the academic medical center commuting to remote campuses. Consultations between community providers and neuroradiologists are thus handled by phone. However, the on-site community body radiologists often field neuroimaging questions. This is typically in the setting of daily medical staff rounds or monthly multidisciplinary conferences. In addition, not infrequently a $\mathrm{TCH}$ community neurologist presents to the reading room, requesting to review imaging. These cases are handled on a case-by-case basis, without a formal protocol. Often the neuroradiologist has already provided a report, and the findings can be easily shown to the provider based on the report description. Other times, it requires a conference call with the neuroradiologist who is interpreting the exam at TCH-TMC.

\section{Challenges and lessons learned}

\section{Faculty development}

Recruitment of radiologists to join the community radiology division has been difficult because the community sites can be viewed as less desirable relative to the academic medical center. Community radiologists typically have limited trainee involvement and access to basic science research. Because promotion has historically been based on education and scholarship, new hires to the community division can be challenged to see how their careers develop and thrive in the community setting. However, there are multiple models in the literature of successful development of hybrid academic-community radiology practices $[3-6,9,12]$.

Our department has created parallel academic and clinical tracks linked to academic/clinical service expectations, amount of academic protected time, and performance-based bonuses, to allow radiologists to self-select their professional priority and interest. These tracks can be chosen independent of division, with faculty in both community and subspecialized divisions including members on both tracks. Our academic affiliate, Baylor College of Medicine, considers clinical productivity along with more traditional measures of academic publication and service leadership when determining academic promotion. The creation of these tracks enables our radiologists to view working at the community hospitals as a different yet equally valued pathway toward professional fulfillment, development and promotion.

Finally, as has been described [13], faculty members participate in a yearly incentive plan. Annually, the incentives are reviewed and sometimes revised. In this process, attention is 
paid to potential opportunity differences to faculty based on a variety of factors, including whether some divisions (such as community) might be unintentionally disadvantaged.

\section{Standardization of imaging protocols and policies}

Assuring uniform imaging protocols and policies, particularly for ultrasound and MRI, is a continual work in progress across the TCH system. Lack of communication for protocol and policy updates is often the root cause for community sites being unaware of changes emanating from the academic center. This has been greatly improved through daily "Morning Huddles" complete with video and audio feed that involve radiology clinical and administrative leaders of all three pediatric hospitals, our community specialty care centers and our adult hospital.

We established protocol governance committees for each modality consisting of physicians, administrators and technologists from all three pediatric hospitals, and these committees meet regularly. From a quality and safety perspective, overlapping roles of the division chiefs for community radiology and radiology quality, as well as campus-specific MR safety officers promote trust, transparency and proper communication.

\section{Equipment}

Standardization of imaging equipment throughout our multiple sites is also a challenge. Using a single vendor for ultrasound and a primary vendor for $\mathrm{CT}$ across all sites greatly simplifies this issue. However, because models and software platforms differ depending upon when equipment was purchased, scheduling challenges for certain exams at particular sites still persist. At one point there were three different MR vendors across all our pediatric hospitals. Factoring additional issues of 1.5-tesla $(\mathrm{T})$ versus 3-T availability and software platform differences between magnets, complete standardization and optimization of MR imaging protocols proved elusive, causing inefficiency and frustration. A concerted effort was made to establish only two MR vendors across the system upon life-cycling existing equipment, and to minimize platform and software variability.

\section{Daily commute}

Perhaps the greatest challenge within our community clinical operational workflow involves the commuting time incurred when physicians, who chose their home location based on working at the academic medical center, are increasingly needed to work at more remote community hospitals. The creation of a dedicated Community Radiology Division mitigated some of the commuting issues by hiring dedicated radiologists who live near their community hospital location. However, academic radiologists still occasionally cover a rotation at the community sites, resulting in job dissatisfaction with one-way commute time not infrequently over an hour (Fig. 1). Current difficulty in recruiting a second interventional radiologist for our community division means that every weekday an academic medical center interventional radiologist drives to one of the two community hospitals.

The trend toward expanding scope of super-specialty imaging services at community sites suggests that a staffing model reliant on radiologists from the academic medical center is not feasible considering the population growth of the Houston metropolitan region and its ever-lengthening commute times. A more sustainable long-term vision is to create conditions at the community site where the radiologists feel valued and fulfilled, allowing them to live in the same community in which they work.

\section{Community call}

As discussed in the "After-hours workflows" section, both body and interventional radiologists are on home pager call for both community hospitals on weekday evenings and nights, and all day on weekends and holidays. These radiologists have no concurrent academic medical center call obligations. If activated overnight, these radiologists still staff their next-day rotations. In the event that emergent cases arise at both community hospitals simultaneously, the after-hours onsite body radiologist at the academic center and Community Radiology Division chief are leveraged for body cases, and the on-call interventional radiologist for the academic center and Interventional Radiology Division chief for interventional cases. To date, we have not needed to use this backup plan.

In 2011, following the initial expansion into TCH-West Campus, interventional and body community call participation was voluntary and was compensated monetarily with a base pager rate as well as hourly activation rates. Call-back volume was low, typically ranging from 0 to 4 cases per month. Based on this caseload and with plans to create a Community Radiology Division, upon opening TCH-The Woodlands in 2017 the body community call was made mandatory and compensation converted to off clinical time based on a certain number of call shifts. To support the three community body radiologists, body radiologists at the academic center also participated, although at less frequency (10 weeks yearly for community division radiologists vs. 10 days yearly for TMC division radiologists). Interventional community call was also converted to mandatory participation, reflecting the growing needs, but continued to receive additional monetary compensation. The number of call activations increased with two maturing community hospitals, currently with 1-10 body call activations per month (average 5.2) and 5-17 interventional call activations per month (average 9.9).

Worsening commute times, mandatory participation of faculty who had never participated in the voluntary system, and 
loss of monetary compensation all contributed to increasing faculty dissatisfaction. Additionally, with increasing volume, call-back frequency increased from 2011. An informal body division survey documented overwhelming dissatisfaction with the community call system. Based on these results, in 2019 hospital leadership reverted the body community call structure back to a voluntary paid system. This has become a faculty satisfier by offering a moonlighting opportunity and without shortage of volunteers.

\section{Potential for disconnectedness}

Maintaining a sense of community among the faculty within the TCH Department of Radiology throughout multiple imaging locations is a priority, but it is challenging. Although we have discussed commuting as a dissatisfier, the staffing of primarily academic-center-based radiologists in the community as well as community radiologists at the medical center does promote a feeling of "one TCH Department of Radiology." Individual faculty members are able to develop an appreciation for site-specific operations, stresses and benefits of the various sites. This broadened perspective is noted during round table discussion at division and departmental faculty meetings where it is clear that the individuals know one another, regardless of whether someone is in the room or calling in from 45 miles away. Allowing the Radiology Department leadership to lead recruitment of community radiologists has ensured a similar standard for the new hires relative to the existing faculty at the academic medical center and has served to extend the culture throughout the system. The difficulty is ensuring that a new hire who mirrors those in the academic medical center is the right fit for a community site.

Despite similar culture, it is easy for the community radiologists to feel occasionally disconnected from their colleagues. Although there is opportunity to interact face-toface with interventional and musculoskeletal radiologists when they rotate to the other sites, they are more frequently alone in the reading room. For all the benefits of video conferencing, they frequently fail or do not provide high-quality audio and video feed, hindering the remote community radiologists' participation and understanding of what is being discussed. At the academic medical center, where the bulk of radiologists are located, it is easy to have curbside discussion of issues and make decisions that are only communicated to the community radiologists weeks or months afterward. However, this loss of connectedness also occurs at the academic medical center, where growth of super-specialty imaging rotations causes interventional, fetal, musculoskeletal, nuclear, cardiovascular and neurologic radiologists to have limited awareness and understanding of their colleagues' daily workflows.
To improve the feeling of connectedness, newly hired community radiologists rotate frequently at the academic medical center for the first 1-2 months so they can learn about our culture and develop relationships. It is our experience, however, that whatever feeling of connectedness is lost with the radiologists at the academic medical center is offset by stronger relationships with community clinical colleagues in the emergency center, surgical and other medical specialties. This is achieved via frequent multi-disciplinary conferences and medical staff meetings as well as social gatherings organized by the community hospital or individuals themselves.

\section{Remote ultrasound exams}

Compared to CT, MRI and radiography, remote staffing of sonography has required the most change to our workflow. Prior to expansion into community sites, all ultrasound exams were checked by the on-site radiologist to assess for quality and need for additional imaging, and to allow radiologists to scan the patient themselves. In the absence of an on-site radiologist, after-hours sonographers at the community hospitals typically discuss the exam over the telephone with the resident, fellow or attending located at TCH-TMC to determine whether additional images are needed prior to discharging the patient. However, there are times (concurrent fluoroscopic procedures, phone calls, face-to-face consultation with providers) when this cannot be accomplished in a timely manner, so we created a workflow to allow for communication between the sonographers and the radiologists via a preliminary note in the PACS communicator. In this scenario, patients with non-stat outpatient exams can be discharged prior to radiologist imaging review. To improve operational flow and support patient satisfaction, sonographers can release stat inpatient or emergency center patients from the radiology department if they have not been able to contact the interpreting radiologist within $15 \mathrm{~min}$. This workflow is also used for sonograms performed at community outpatient clinics during normal weekday operating hours.

The lack of on-site radiologists does have some disadvantages regarding community ultrasound workflows. The most typical outcome is that the patient is asked to return for repeat imaging when a radiologist is on-site, at no charge to the patient. Unsurprisingly, this can lead to patient dissatisfaction. In response, the sonographers have been encouraged to contact the radiologist for any difficult cases.

Finally, with the heterogeneity introduced by multiple sites and personnel, the need for sonographer quality control has increased. To this end, a specific ultrasound manager position has been created for the TCH system. In addition, before sonographers are approved to staff off-site locations alone, they must complete a required curriculum and a minimum number of cases proctored by radiologists. 
Table 2 Rapid expansion of Texas Children's Hospital (TCH)West Campus Radiology

Department, 2011-2020

\begin{tabular}{ll}
\hline 2011 & 2020 \\
\hline $\begin{array}{l}5 \text { staff, including technologists and } \\
\text { administrative support }\end{array}$ & $\begin{array}{c}56 \text { staff, including PACU nursing in addition to } \\
\text { technologists and administrative support }\end{array}$ \\
2 radiography suites & 4 radiography suites \\
2 portable radiography units & 5 portable radiography units \\
1 C-arm & 2 C-arms \\
1 MRI (1.5 T) & 2 MRIs (1.5 T and 3 T) \\
1 CT & 1 CT (upgraded to expand scope for cardiac imaging) \\
1 ultrasound unit & 3 ultrasound units \\
No interventional radiology services & 1 interventional radiology suite \\
\hline
\end{tabular}

$P A C U$ post-anesthesia care unit

\section{Remote consultations}

Frequently, an imaging study is interpreted by a radiologist who is not physically present at the same campus as the referring provider. This occurs for all community neuroradiology cases and many community super-specialty cases, and can be a communication challenge after hours when the interpreting radiologists are only at TCH-TMC. Video conferencing capabilities are used for scheduled cross-campus multi-disciplinary imaging conferences but rarely for one-on-one imaging consultation. A significant percentage of new clinical hires at the community sites are advanced practice providers (e.g., physician assistants, nurse practitioners) and junior attending physicians who are not known to the majority of the radiologists, the bulk of whom have spent the majority of their time at the academic medical center.

Cultural differences, lack of familiarity with the clinical care team at the community hospitals, and the lack of faceto-face contact during telephone discussion can contribute to misunderstandings and impede delivery of critical clinical information between facilities. However, the system has taken steps to improve communications. Specifically, at the community hospitals, scheduled thrice weekly care conferences promote face-to-face discussions. Video conferencing capabilities continue to be upgraded. Additionally, there are daily scheduled systemwide huddles, where personnel from all service lines and sites are represented. Combined with systemwide initiatives to promote a culture of belonging, no matter where an associate is based, a sense of trust in one's colleagues, despite face-to-face interaction, has been developed.

\section{Opportunities}

\section{Expansion of academic super-specialty sections}

While imaging volumes have continued to increase at our primary academic hospital, the bulk of increasing imaging volumes for the TCH system has been generated by growth at our community locations (Table 2, Fig. 3). In addition to the creation of the Community Radiology Division, the growth in the community has fueled expansion of existing academic sections as well as the hiring of overnight in-house Emergency Department pediatric radiologists. Creation or expansion of the fetal, musculoskeletal, cardiovascular and nuclear radiology sections was achieved via the additional hires needed to support the growing system imaging volume. Having 28 radiologists in 2010, we now have 43 radiologists.
Fig. 3 Texas Children's Hospital (TCH) Radiology Enterprise imaging volumes. In 2019 the cumulative imaging volumes from the two community hospitals, specialty care and urgent care centers approximated to volumes at the academic medical center. Vertical bars designate opening dates of the community hospitals for inpatient services

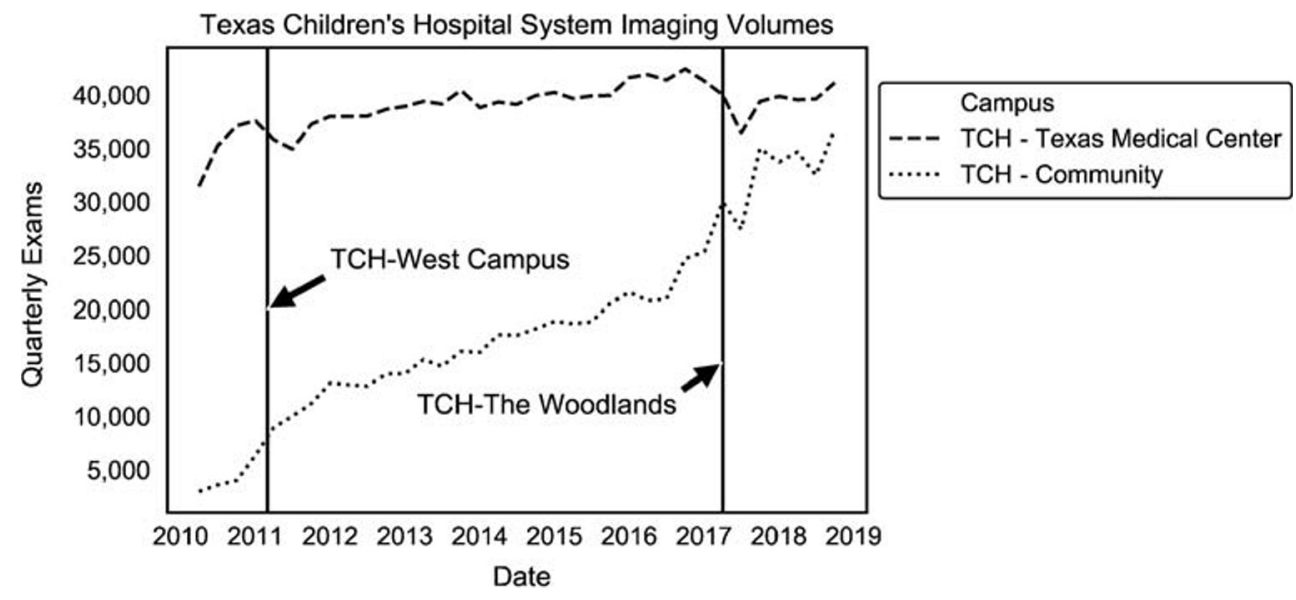


While economies of scale have undoubtedly subsidized the faculty growth, improved imaging volume alone does not fully account for the increased revenue stream. Rather, expansion into the community sites has been favorable for reimbursement because of a variety of factors, including payer mix. This increased revenue stream from the community practice helps support the academic missions of the overall Radiology Department, helping ensure protected time for administrative, educational and research work. In addition to allowing a greater proportion of our academic radiologists to practice superspecialty imaging, the increased staffing has led to a decrease in the overall call frequency.

\section{Improved quality of life}

Although community hospitals vary, a common trait that defines them is their sense of community and the greater interconnectedness among clinical care teams, administrators and patients that enables them to stand out from academic medical centers [14]. Working as a generalist and being able to devote $100 \%$ effort to clinical work is often a welcome respite from juggling multiple responsibilities of clinical work, education, research and administration. With rising costs of real estate close to the academic medical center, fewer academic radiologists are able or willing to assume the financial burden that would allow a short commute. In contrast, our TCH-West Campus and TCH-The Woodlands community hospitals are located in some of the most desirable neighborhoods in the region, with affordable housing, low cost of living, and the promise of a short commute. Additionally, community call moonlighting opportunities have been extremely popular as a way of earning additional income.

\section{Unique training opportunities}

One of the most important missions of academic radiology is to train the next generation of radiologists. Although a significant proportion of radiologists, both adult and pediatric, practice in a community setting, most radiology residents spend little time during residency working in the community [6]. Body radiology rotations are available to our pediatric radiology learners at TCH-West Campus. This rotation is unique within our training system because it allows students to handle a higher volume of more often straightforward imaging cases. Fluoroscopy volume is decreasing at many academic medical centers but is still widely utilized at community-based outpatient sites, and participation in community rotations enhances development of this skill set. Additionally, this rotation can help trainees make better informed decisions regarding choosing community, academic or blended pathways for their careers [6]. Finally, though the proportion of exams performed at the community sites has rapidly increased, volume at the medical center, which is the main site for trainees, has also continued to increase, though at a slower rate. Consequently, while there was concern that shift to community sites could detract from educational opportunities, we have not seen this in the volume of studies available for trainees to interpret.

\section{Improved patient satisfaction}

In a 2009 survey, $78 \%$ of consumers stated they would not pay a higher premium simply for access to an academic medical center, particularly if similar services were available in their community $[9,14]$. The location of high-quality pediatric imaging services throughout the Houston metropolitan region and specifically at two community hospitals allows patients and their families to choose between receiving equally highquality diagnostic care at a large quaternary hospital where the sickest of the sick are cared for and where they are often pressed with parking and wayfinding issues, versus a smaller, less threatening and often friendlier community hospital [14]. This choice empowers patients and is often credited for higher overall patient satisfaction scores at our community hospitals relative to the academic medical center (Internal data, Press Ganey surveys).

\section{Conclusion}

Development and execution of a pediatric community imaging strategy has improved access to high-quality diagnostic and therapeutic care to many in the greater Houston metropolitan region. The increased pediatric community imaging volume and subsequent revenue has brought both challenges and benefits to the TCH Pediatric Radiology Department. Although we report the experiences from a single large quaternary pediatric health care system and many experiences and workflows might not be transferable to other institutions, this manuscript can serve as an experience and reflection document.

\section{Compliance with ethical standards}

Conflicts of interest None

\section{References}

1. Fairchild D, Wesslund R (2018) Collaborating for value: the path to successful academic-community relationships. Healthcare Financial Management Association, Westchester

2. Natesan R, Yang WT, Tannir H, Parikh J (2016) Strategic expansion models in academic radiology. J Am Coll Radiol 13:329-334

3. Croft CR, Dial R, Doyle G et al (2016) Integrating a community hospital-based radiology department with an academic medical center. J Am Coll Radiol 13:300-302.e306 
4. Gusenbauer KL, Patlas MN, Kielar AZ, Katz DS (2018) Recipe for a successful hybrid academic-community radiology practice: Canadian experience. Acad Radiol 25:240-243

5. Kalambo M, Parikh JR (2018) The community-based academic radiologist. J Am Coll Radiol 15:1500-1504

6. Patlas MN, Farshait N, Duszak R Jr (2017) Academic and community radiology practice integration: the Canadian experience. J Am Coll Radiol 14:1124-1126

7. Spalluto LB, Thomas D, Beard KR et al (2019) A communityacademic partnership to reduce health care disparities in diagnostic imaging. J Am Coll Radiol 16:649-656

8. Sussman AJ, Otten JR, Goldszer RC et al (2005) Integration of an academic medical center and a community hospital: the Brigham and Women's/Faulkner hospital experience. Acad Med 80:253-260

9. Fleishon HB, Itri JN, Boland GW, Duszak R Jr (2017) Academic medical centers and community hospitals integration: trends and strategies. J Am Coll Radiol 14:45-51
10. Nuckols T, Weingarten S, Priselac T (2019) What value-based payment means for academic medical centers. NEJM Catalyst, Waltham

11. Parikh JR, Brown J, Yang WT, Tannir H (2017) Network collaboration of an academic institution and a community health organization. J Am Coll Radiol 14:282-285

12. Boyse TD, Paulson EK (2018) Creating a community radiology division: an academic radiology department's 3-year experience. J Am Coll Radiol 15:1651-1654

13. Bisset GS 3rd (2017) An honest day's work: pay for performance in a pediatric radiology department. Pediatr Radiol 47:798-802

14. (2015) The modern definition of a community hospital. Becker's Hospital Review, Chicago

Publisher's note Springer Nature remains neutral with regard to jurisdictional claims in published maps and institutional affiliations. 